\title{
TERAPIAS UTILIZADAS EM PACIENTES DIAGNOSTICADAS COM CÂNCER DE MAMA EM UMA CIDADE DO SUL DE MINAS GERAIS
}

\author{
Karinne Naara Matos de BARROS ${ }^{1}$ \\ Lara Miranda Rodrigues da CUNHA ${ }^{1}$ \\ Roberta Bessa Veloso SILVA ${ }^{2}$ \\ Gérsika Bitencourt SANTOS ${ }^{2}$
}

1. Acadêmicas de Medicina da Universidade José do Rosário Vellano.

2. Docentes da Universidade José do Rosário Vellano.

Universidade José do Rosário Vellano - UNIFENAS, Alfenas-MG.

E-mail: gersika.santos@unifenas.br

Recebido em: 11/02/2016 - Aprovado em: 26/05/2016 - Disponibilizado em: 30/07/2016

\begin{abstract}
Resumo
Introdução: Segundo o Instituto Nacional de Câncer José Alencar Gomes da Silva (INCA), o câncer de mama é o mais comum tipo de câncer entre as mulheres. Os tratamentos do câncer mamário podem ser classificados em: Terapia Local, Terapia Sistêmica, Terapia Adjuvante e Terapia Neoadjuvante. Objetivos: O estudo visa conhecer as terapias farmacológicas e não farmacológicas realizadas em pacientes diagnosticados com câncer de mama em uma cidade do sul de Minas Gerais. Método: Foram aplicados 34 questionários a pacientes com câncer de mama atendidas na Santa Casa de Misericórdia de Alfenas, MG. As variáveis coletadas foram: medicamentos ou outro tipo de tratamento utilizado para pacientes com câncer de mama, período em que foi diagnosticada a doença, possíveis efeitos colaterais obtidos pela paciente e a adesão ao tratamento realizado. Resultado: O câncer de mama é normalmente diagnosticado na pré e pós-menopausa. Os tratamentos mais realizados são a quimioterapia, seguida da terapia hormonal, radioterapia e mastectomia.Dentro da terapia hormonal, o medicamento mais utilizado é o Tamoxifeno. Conclusão: Observa-se que grande parte das mulheres possui algum efeito colateral referente às terapias mais invasivas - Quimioterapia e Terapia Hormonal.

Palavras-Chave: Câncer de Mama. Quimioterapia. Radioterapia.Mastectomia. Tamoxifeno.
\end{abstract}

\begin{abstract}
Introduction: According to the National Cancer Institute José Alencar Gomes da Silva (INCA), breast cancer is the most common type of cancer among women. of breast cancer treatments can be classified into : Local therapy , systemic therapy, adjuvant therapy and therapy Neoadjuvant.Objectives: The study aims to know the pharmacological therapies and nonpharmacological performed in patients diagnosed with breast cancer in a town in southern Minas Gerais. They were applied 34 questionnaires to patients with breast cancer treated at the Santa Casa de Misericordia de Alfenas, MG.Methods: The variables collected were: medication or other treatment used for patients with breast cancer , during which it was diagnosed the disease, possible side effects obtained from the patient and adherence to the treatment performed. Result:Breast cancer is usually diagnosed in pre- and postmenopausal women. The most performed treatments are chemotherapy, followed by hormone therapy, radiation therapy and mastectomy. Within the hormone therapy, the most widely used drug is Tamoxifen. Conclusion:It is observed that most women have some side effects related to more invasive therapies - Chemotherapy and Hormone Therapy.
\end{abstract}

Kewords: Breast Cancer. Chemotherapy.Radiotherapy.Mastectomy. Tamoxifen.

\section{INTRODUÇÃO}

Segundo o Instituto Nacional de

Câncer José Alencar Gomes da Silva (INCA),

o câncer de mama é o mais comum entre as mulheres, respondendo por $22 \%$ dos casos novos a cada ano, levando em consideração que o Brasil ainda é um país com altas taxas de mortalidade, devido ao fato da doença 
ainda ser diagnosticada em estádios avançados.

De acordo com o Hospital de Câncer de Barretos, oauto-exame das mamas, hoje em dia, deve ser chamado de auto-cuidado, e pode ser feito pelo menos uma vez ao mês a partir da adolescência, preferencialmente no mesmo dia do mês para que as mulheres se familiarizem com suas mamas. Após os 40 anos, a mamografia começa a ser um exame importante para a detecção da doença e recomenda-se que seja feito pelo menos uma vez por ano a partir daí. Todas as mulheres deveriam procurar um mastologista para acompanhamento e exame anual durante sua vida, mas principalmente a partir dos 40 anos.

Geralmente o câncer de mama não apresenta sintomas no início. A partir do momento que começa a ser palpável, pode estar associado a um caroço na mama. Também pode ser representado por áreas de abaulamentos ou retrações de pele. Manchas ou alterações na pele da mama. Ainda pode estar ligado com saída de líquido do bico da mama, geralmente no caso do câncer estes líquidos são sanguinolentos ou semelhantes à cor da água de geladeira ao descongelarmos o congelador.

Entre os tratamentos disponíveis para o câncer de mama, o Instituto Oncoguia (2014) os classificam em terapia local, como por exemplo a cirurgia e a radioterapia; terapia sistêmica, que consiste na quimioterapia, terapia hormonal e terapiaalvo, visando atingir as células cancerosas em qualquer parte do corpo; e terapia adjuvante que tem como objetivo destruir as células neoplásicas remanescentes. Caso seja necessário reduzir o tumor e permitir uma cirurgia menos invasiva, recorre-se à terapia neoadjuvante, utilizando-se a quimioterapia ou terapia hormonal antes da realização da cirurgia.

Entre os fármacosquimiopreventivos, encontra-se o tamoxifeno. Esse medicamento pode ser utilizado em pacientes na pré e pósmenopausa e age ligando-se ao receptor tumoral para estradiol, competindo, assim, com esse hormônio. O tamoxifeno possui ação complexa, uma vez que, em tecido mamário, apresenta um efeito antagônico ao estrogênio, mas pode exibir um efeito agonista parcial ao estrogênio, o que pode ser benéfico, prevenindo a desmineralização óssea em mulheres após a menopausa; ou maléfico, aumentando o risco de câncer de endométrio e causando fenômenos tromboembólicos (LEITE, Costa MarabottiFranciéleet al, 2010).

Sinais e sintomas como náuseas, vômitos, fadiga, alopécia, mucosite, alterações na pele e complicações como infecções, neuropatia periférica, neutropenia febril, toxicidade renal, hepática e disfunção reprodutiva são alguns efeitos colaterais causados pela quimioterapia, não excluindo as alterações emocionais que podem contribuir até mesmo para desistência do tratamento (GONÇALVES, Leila Luíza et al, 2009). 
Esse estudo, portanto, tem como objetivo conhecer as terapias farmacológicas e não farmacológicas realizadas em pacientes diagnosticados com câncer de mama em uma cidade do sul de Minas Gerais, bem como analisar quais os efeitos colaterais frequentemente relatados e como esse dado se relaciona com a adesão ao tratamento.

\section{MÉTODO}

A pesquisa foi realizada no município de Alfenas, Minas Gerais, o qual apresenta de acordo com o censo IBGE de 2015, uma população de 78.712 habitantes. A população amostral foi as pacientes com câncer de mama, independentemente do tipo de tratamento administrado.

As entrevistas foram realizadas com as pacientes diagnosticadas com câncer de mama cadastradas no Hospital do Câncer da Santa Casa de Alfenas. 34 mulheres foramesclarecidas sobre o projeto realizado e convidadas a participar de um questionário, incluindo a coleta de dados de acordo com o tratamento que elas fazem uso, além de seus dados socioeconômicos. Contudo, foram informações restritas aos pesquisadores responsáveis, portanto, os entrevistados não terão sua identidade exposta no estudo.

As variáveis coletadas foram: medicamentos ou outro tipo de tratamento utilizado para pacientes com câncer de mama, período em que foi diagnosticada a doença, tempo de utilização da terapia, possíveis efeitos colaterais obtidos pelo paciente de acordo com a terapia submetida e a adesão ao tratamento realizado.

Foram estimadas as prevalências de diferentes tipos de tratamento de câncer de mama, considerando que as terapias foram categorizadas em farmacológicas ou não farmacológicas.

O presente trabalho foi aprovado pelo Comitê de Ética da Unifenas, sob parecer de número 951.055.

\section{RESULTADOS E DISCUSSÃO}

Os resultados deste estudo mostram que o câncer de mama é normalmente diagnosticado na pré-menopausa e pós menopausa.

De acordo com Lúcia Cecília da Silva (2008), o câncer de mama é relativamente raro antes dos 35 anos de idade, mas acima desta faixa etária sua incidência cresce rápida e progressivamente. As estatísticas indicam o aumento de sua frequência tanto nos países desenvolvidos quanto naqueles em desenvolvimento. Segundo a Organização Mundial da Saúde (OMS), nas décadas de 60 e 70 registrou-se um aumento de 10 vezes nas taxas de incidência ajustadas por idade nos Registros de Câncer de Base Populacional de diversos continentes (INCA, 2006a). No Brasil, é a primeira causa de morte por câncer na população feminina, principalmente na faixa etária entre 40 e 69 anos (INCA, 2006b). Em países do Ocidente, entre todas as causas de óbito, ele é a mais comum em 
mulheres abaixo da idade de 50 anos (Boyd, 1999).

O câncer atualmente é tratado como uma terapia sistêmica, portanto, muitas vezes, o tratamento cirúrgico é seguido de uma terapia adjuvante. São estas: radioterapia, que constitui-se da utilização raios de alta energia que têm a capacidade de destruir as células cancerosas e impedir que elas se multipliquem. É um tratamento local, sendo realizado de forma externa ou interna; quimioterapia, utilização de drogas que agem na destruição das células malignas. Pode ser aplicada através de injeções intramusculares , endovenosas ou por via oral; hormonioterapia, que tem como finalidade impedir que as células malignas continuem a receber o hormônio que estimula seu crescimento. Esse tratamento pode incluir cirurgia que remova os ovários - órgãos responsáveis pela produção desses hormônios (MAKLUF; DIAS; BARRA, 2006 apud RAMOS; LUSTOSA, 2009, p.91).

Os tratamentos mais realizados relatados nesse estudo são a quimioterapia $(41,25 \%), \quad$ seguida da terapia hormonal(21,25\%), radioterapia $(20,0 \%)$ e mastectomia $(17,5 \%)$, como mostrado no Gráfico 1.
Gráfico 1 - Tipos de tratamentos realizados pelas pacientes portadoras de

câncer de mama.

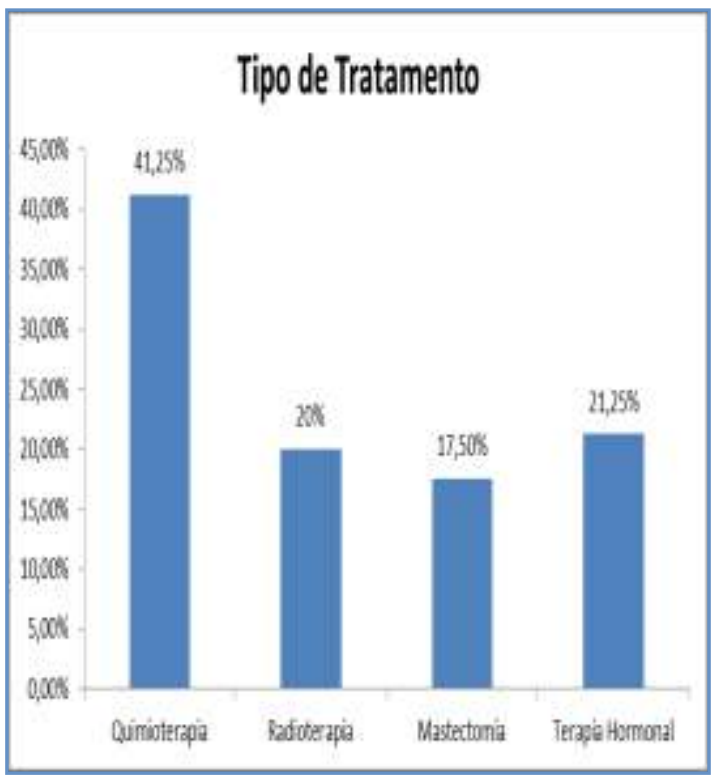

Fonte -Elaborada pelo autor.

Deve-se ressaltar que na terapia hormonal, $70,6 \%$ das pacientes fazem tratamento com o tamoxifenoacompanhado ou não de outros medicamentos (Gráfico 2).

Gráfico 2 - Prevalência do uso de Tamoxifeno na terapia hormonal de mulheres

com câncer de mama.

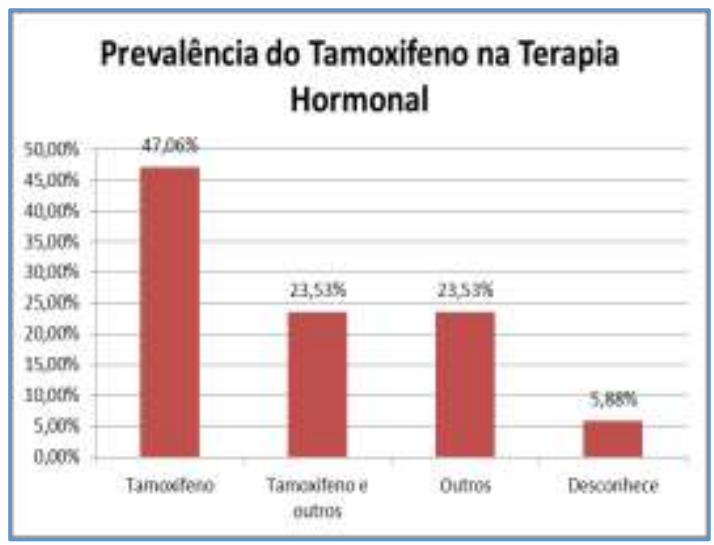

Fonte - Elaborada pelo autor.

O uso de tamoxifeno pode associar-se ao aparecimento ou à piora das ondas de calor. Vale ressaltar que outros sintomas 
como artralgia, ganho de peso, alterações do humor e dificuldades de concentração são mais prováveis de relacionarem-se ao processo de envelhecimento e à menopausa do que ao tamoxifeno (CONDE, Marques Délioet al, 2006).

De acordo com Nicolussi e Sawada (2011), apesar de ter aumentado a sobrevida, as terapias sistêmicas como a quimioterapia, bem como as terapias hormonais adjuvantes influenciaram negativamente a Qualidade de Vida Relacionada à Saúde, devido aos efeitos adversos causados.

Como se pode ver no Gráfico 3, em relação aos efeitos colaterais, pode-se observar que o mais frequentemente relatado pelas 16 mulheres que foram submetidas somente à quimioterapia foram enjôos $(56,25 \%)$ e quanto às 17 mulheres submetidas à quimioterapia associada à terapia hormonal foram relatados enjôos $(35,29 \%)$ seguidos de ondas de calor $(23,52 \%)$.

Gráfico 3 -Percentual dos efeitos colaterais relatados pelas pacientes que se trataram com a quimioterapia e/ou a terapia hormonal.

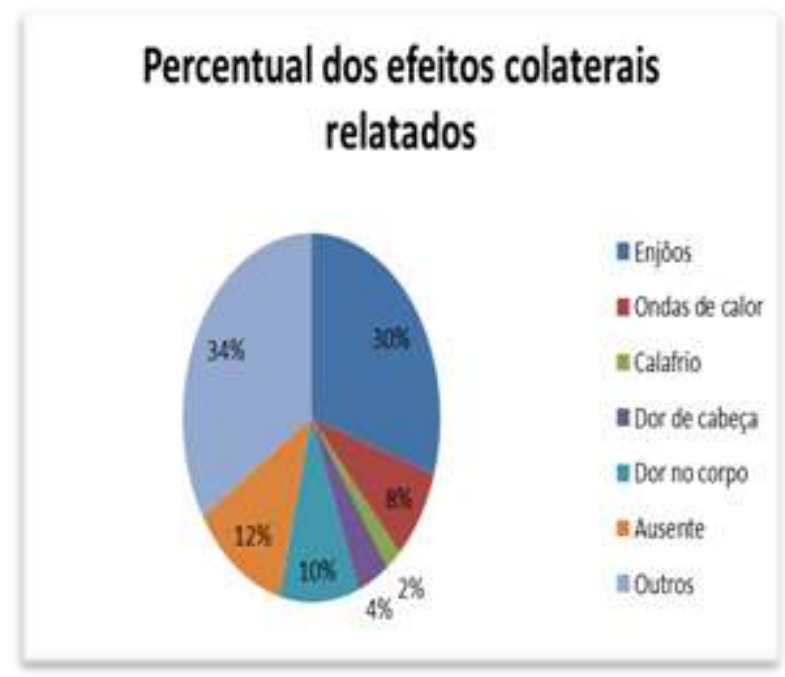

Fonte - Elaborada pelo autor.
As relações sistemáticas entre bemestar físico e funcional e depressão e entre bem-estar funcional e ansiedade levam a pensar que a correção dos sintomas decorrentes dos efeitos colaterais do diagnóstico, tratamento cirúrgico e estado psicológico da paciente deva ser realizada de maneira eficiente.

Dos resultados referentes à adesão ao tratamento, observou-se que das 34 pacientes, 30 fazem uso de medicamento diário, das quais $93,33 \%$ nunca se esqueceram de tomálo e $6,66 \%$ se esquecem uma vez na semana. Quanto aos medicamentos que não são de uso contínuo, utilizados por apenas 18 pacientes entrevistadas, $88,88 \%$ apresentam uma assiduidade classificada como muito boa, enquanto que 5,55\% apresentam assiduidade boa e 5,55\% assiduidade ruim.

Contrapondo-se ao nosso estudo, um dos aspectos encontrados na literatura que justifica a baixa adesão é a duração do tratamento, que muitas vezes é difícil de ser seguido de forma regular. Além disso, não se pode esquecer de que fatores como o sistema de saúde, a prestação de serviços assim como a doença e seu tratamento, também influenciam a adesão. Esse problema acaba sendo ignorado pelos profissionais de saúde, sendo a baixa adesão normalmente a primeira razão para a piora do quadro clínico (SOUZA, Bianca Fresche de et al, 2013). 


\section{CONCLUSÃO}

Observou-se que grande parte das mulheres possui algum efeito colateral referente às terapias mais invasivas, de certa forma, sendo um quadro clínico normal e esperado para o tratamento do câncer de mama. Demonstra-se, portanto, a relevância dessa pesquisa, pois surge a oportunidade de pontuar efeitos colaterais de medicamentos que podem ser aperfeiçoados pela indústria farmacêutica na tentativa de melhorar a qualidade de vida das pacientes tratadas. Assim como, sugere a importância de um acompanhamento multidisciplinar na área hospitalar, o que pode conscientizar e auxiliar essas mulheres na aderência ao tratamento e no seu bem estar físico e emocional.

Sobre a Terapia Hormonal, sobressaise, em grande prevalência, o uso do Tamoxifeno,o qual tem sido a droga mais utilizada para o tratamento desta doença sendo que seu uso está indicado em casos de metástase, assim como no tratamento adjuvante (pós-operatório) de pacientes com tumor em fase inicial e avançada, em casos onde o paciente possui linfonodos acometidos ou livres de tal doença, e em casos de pacientes com carcinoma ductal in situ.

Em relação ao efeito colateral da terapia hormonal, o mais apontado foi a constante presença de enjôos (náuseas e vômitos), seguido de ondas de calor. Com isso, deve-se alertar às pacientes que apresentam a doença na pré-menopausa, que esses sinais e sintomas podem ser confundidos com o climatério.

\section{REFERÊNCIAS}

AVELAR, Anne Melina Ambrósio et al. Qualidade de vida, ansiedade e depressão em mulheres com cancêr de mama antes e após a cirurgia. Revista de Ciências

Médicas, Campinas, v. 15, n. 1, p.11-20, 2006.

BARRETOS, Hospital de câncer. Prevenção e Sintomas do Câncer de Mama. Disponível em: <

http://www.hcancerbarretos.com.br/cancerde-mama/92-paciente/tipos-de-cancer/cancerde-mama/162-prevencao-e-sintomas-docancer-de-mama>. Acesso em: 10 fev. 2016.

CONDE, Délio Marques et al . Qualidade de vida de mulheres com câncer de mama.Rev. Bras. Ginecol. Obstet., Rio de Janeiro, v. 28, n. 3, p. 195-204, Mar. 2006.

GONÇALVES, L. L. C et al. Mulheres com câncer de mama: ações de autocuidado durante a quimioterapia. Revista

Enfermagem UERJ, Rio de Janeiro, v. 17, n. 4, p. 575-580, out./dez. 2009.

LEITE, FranciéleMarabotti Costa et al. Mulheres com Diagnóstico de Câncer de Mama em Tratamento com Tamoxifeno: Perfil Sociodemográfico e Clínico. Revista Brasileira de Cancerologia, Rio de Janeiro, v. 1, n. 57, p.15-21, jan. 2011.

NICOLUSSI, Adriana Cristina; SAWADA, NamieOkino. Qualidade de vida de pacientes com câncer de mama em terapia adjuvante. Rev. Gaúcha Enferm., Porto Alegre, v. 32, n. 4, p. 759-766, Dec. 2011

ONCOGUIA, Equipe. Tratamentos do Câncer de Mama. Disponível em: <http://www.oncoguia.org.br/conteudo/tratam entos/15/12/>. Acesso em: 10 fev. 2016.

RAMOS, Bianca Figueiredo; LUSTOSA, Maria Alice. Câncer de mama feminino e 
psicologia. Rev. SBPH, Rio de Janeiro, v. 12, n. 1, p. 85-97, jun. 2009

SILVA, Lucia Cecilia da. Câncer de mama e sofrimento psicológico: aspectos relacionados ao feminino. Psicol. estud., Maringá, v. 13, n. 2, p. 231-237, June 2008 .

SOUZA, Bianca Fresche de et al . Pacientes em uso de quimioterápicos: depressão e adesão ao tratamento. Rev. esc. enferm. USP, São Paulo, v. 47, n. 1, p. 61-68, Feb. 2013 